\title{
Entornos virtuales como espacios de enseñanza-aprendizaje. "Un enfoque teórico para la educación superior"
}

\author{
Virtual environments as teaching-learning spaces. "A theoretical approach for higher education
}

Ambientes virtuais como espaços de ensino-aprendizagem. "Uma abordagem teórica do ensino superior"

\author{
Maritza Villacis Lizano \\ maritzavillacis@uti.edu.ec \\ https://orcid.org/0000-0001-5935-707X
}

Universidad Indoamérica, Quito-Ecuador

\author{
Mayra Moreno Genovés \\ mayramoreno@uti.edu.ec \\ https://orcid.org/0000-0002-8645-1296
}

Universidad Indoamérica, Quito-Ecuador

\author{
Raul Benavides Lara \\ raul.benavides@iespoch.edu.ec \\ https://orcid.org/0000-0001-5560-845X
}

Escuela Superior Politécnica de Chimborazo, Riobamba-Ecuador

Recibido 28 de mayo 2021 | Arbitrado y aceptado 11 de junio 2021 | Publicado en 01 julio 2021

\begin{abstract}
El artículo muestra un modelo teórico que permite estudiar el potencial convertidor de los entornos virtuales a partir de su capacidad para mediar las relaciones entre docentes, estudiantes y el contenido; esta orientado desde una representación constructivista socio cultural, y basado en los elementos básicos de un modelo teórico. Se analizan algunos temas puntuales concernientes al estudio de los entornos virtuales, y se plantea un sustento metodológico cualitativo en donde se aplica un modelo de cinco fases la cuales son: preparatoria, descriptiva, interpretativa por núcleo temático, de construcción teórico global y de extensión para su análisis de estudio. Finalmente, se enfatizan aspectos concernientes a la contribución de lo virtual en la educación, ideados como espacios para el progreso de técnicas de enseñanza y aprendizaje.
\end{abstract}

Palabras clave: Enfoque; fases; enseñanza; entornos; espacio; virtuales
RESUMEN

The article introduces a theoretical model to study the converting potential of virtual environments based on their ability for facilitating the relationships between teachers, students, and content. Also, it is focused on a socio-cultural constructivist representation and is based on the fundamental elements of the theoretical model. The study analyzes some specific issues concerning the study of virtual environments and proposes qualitative methodological support where a five-phase model is applied: preparatory, descriptive, interpretative for thematic nucleus, global theoretical construction, and extension for its study analysis. Finally, aspects concerning the contribution of the virtual in education are emphasized, designed as spaces for the progress of teaching and learning techniques.

Key words: Approach; phases; teaching; environments; space; virtual

\section{RESUMO}

$\mathrm{O}$ artigo mostra um modelo teórico que permite estudar o potencial de conversão dos ambientes virtuais a partir de sua capacidade de mediar as relações entre professores, alunos e conteúdos; é orientado a partir de uma representação sócio-cultural construtivista, e baseado nos elementos básicos de um modelo teórico. Algumas questões específicas relativas ao estudo de ambientes virtuais são analisadas, e um apoio metodológico qualitativo é proposto, no qual um modelo de cinco fases é aplicado: preparatório, descritivo, interpretativo por núcleo temático, construção teórica global e extensão para a análise do estudo. Finalmente, aspectos relativos à contribuição do virtual na educação, concebidos como espaços para o progresso das técnicas de ensino e aprendizagem, são enfatizados.

Palavras-chave: Abordagem; fases; ensino; ambientes; espaço; virtual; espaço; virtual 


\section{INTRODUCCIÓN}

Con la integración de las tecnologías en los métodos de enseñanza aprendizaje, la educación tiende a desplegarse como un sistema abierto y continuo que demanda la innovación de orientaciones pedagógicas modernas para beneficiar el estudio autónomo e independiente, el trabajo en conjunto, el desenvolvimiento de técnicas interactivas de comunicación y apropiación del conocimiento, influidos por la acción dialógica entre docentes y estudiantes, así como por el uso de nuevas tecnologías de la información y las comunicaciones.

Las investigaciones analizadas señalan que, el aprendizaje activo aguantados en el extenso contexto de las TIC y en el diseño de nuevos espacios de aprendizaje con el uso óptimo de esos recursos, establecen un indicio necesario para conseguir el pleno camino de los entornos virtuales en todas las modalidades de estudio, además se debe avalar el control del aprendizaje por parte del propio estudiante.

Las instituciones educativas deben prepararse para enfrentar los grandes desafíos científico-tecnológicos de la globalización y las comunicaciones. La sociedad actual, llamada por algunos "sociedad del conocimiento", manifiestan la importancia progresiva de las Tecnologías de la Información y las Comunicaciones (TIC) como fuente para el adelanto. Las TIC han incrustado cambios en la educación de modo particular, que han impactado de manera apresurada al tomar en consideración que los estudiantes del siglo XXI tienen que desenvolverse en un ambiente rico en investigación y, unido a ello, "desplegar dos competencias adicionales, además de las clásicas (conocer, hacer y ser): deben aprender a buscar la información y deben saber aplicar o convertir esta en función de solucionar nuevos problemas". (Nobile y Luna, 2015).
Los primeros estudios en torno al tema de las TIC y entornos virtuales de enseñanza revelan una importancia para los investigadores en los últimos treinta años. Se destacan, desde la década de los años ochenta del pasado siglo, en el plano internacional, los estudios de Chadwick y Vaquero (1987); y más próximos en el tiempo son los de Marqués (2002, 2004) citado por León y Moracén (2015) en los que se recalca la necesidad de manejar las TIC's desde un enfoque didáctico y pedagógico.

Para Fernández y Arroyo (2018) la educación superior en Ecuador ha experimentado grandes cambios y nuevos requerimientos. Contempla que los programas conseguirán ser en modalidad de estudios presencial, semipresencial, a distancia virtual, en línea y otros. El aprendizaje es beneficiado por el manejo de tecnologías informáticas y entornos virtuales que fortalecen la dinámica e interacción entre docentes y educandos, sin las limitaciones del tiempo que ocurren en la modalidad presencial directa. El soporte tecnológico más empleado para la creación de entornos virtuales de aprendizaje se denomina Moodle. El software admite el proceso de sistemas de gestión de la enseñanza y beneficia el diseño de cursos en línea participativos.

\section{Los entornos virtuales de enseñanza y aprendizaje}

Para establecer conceptualmente lo que es un Entorno Virtual de Aprendizaje (EVA) se cita a Nóbile (2015) quien recopila varias conceptualizaciones concerniente de entorno virtual de enseñanza y aprendizaje, por ejemplo, en términos frecuentes expresa que es un sistema informático perfeccionado con fines didácticos, es decir, proyecta un fin pedagógico; así mismo, se profundiza en la función que este tiene el cual puede representarse como el espacio corporal donde un estudiante o colectividad de estudiantes despliegan su tarea, incluyendo todos los instrumentos, 
escritos y otros compendios que logran ser hallados en dichos contextos, pero asimismo las particularidades socio/culturales para tal trabajo.

Una conceptualización atrayente es dada por León (2015), quien menciona que los entornos virtuales son áreas de notificación que admiten el cambio de indagación y que harían viable, según su uso, la realización de un contenido de enseñanza en el cual se facilitará la cooperación de docentes y estudiantes, en un cuadro de cooperación, a través de unos temas culturalmente elegidos y plasmados mediante la representación de distintos idiomas que el contorno tecnológico es apto de llevar.

Para Sánchez y Salvador (2010) en su estudio de las TIC y su incidencia en la esfera de la educación escolar, proyectan una doble entrada: la primera se fundamenta en cómo estas tecnologías consiguen ser manejadas con beneficio según sus particularidades, para originar el aprendizaje; la segunda, en cómo la anexión de las TIC a la educación y los usos que se crean de ellas consiguen llegar a soportar una transformación fundamental de los ambientes de enseñanza y aprendizaje. En lo que resta, nos concentraremos en examinar rápidamente cómo las TIC convierten o cambian los entornos de educación formal y cómo se han transformado en un elemento clave para la aparición de nuevos contextos educativos incorporados a entornos virtuales o en línea.

Un EVA tiene como particularidad convertirse en un espacio que facilite la transformación de las modalidades de enseñanza en los diferentes niveles (Cedeño, 2019); para este estudio, se ha establecido el impacto que ha obtenido la realización de este tipo de contextos en los estudiantes universitarios. Una de las particularidades de los ambientes virtuales de aprendizaje es que cuenta con funcionalidades que admiten la comunicación clara y activa entre los actores del proceso, originando nuevos roles para el docente, que se convierte en un guía y moderador, y para los estudiantes, con un papel más dinámico en la cimentación de los conocimientos.

Esto es viable lograrlo porque estas áreas ofrecen sitios de trabajo y colaboración para equipos de Investigación. Hoy por hoy se genera la necesidad de localizar la manera de instaurar herramientas pedagógicas vinculadas con los contextos virtuales de aprendizaje de tal modo que se cree interacción entre el conocimiento técnico y el pedagógico. En definitiva, se busca con ello innovar la educación tradicional con el apoyo de la tecnología, pero sin perder la calidez de la interacción social, el aspecto crítico y el uso procedente de contextos educativos. De esta manera es viable aspirar a la investigación del conocimiento individual a través de un aprendizaje de carácter autónomo y colaborativo, en el que se desplieguen cambios profundos que se acomoden a medida que la tecnología avanza.

Para Sánchez (2010) una de las determinaciones de los entornos virtuales es crear, examinar y percibir las ordenaciones de entornos para la enseñanza y el aprendizaje en línea el cual implica esencialmente, examinar su enorme complicación interior, agrupada a la escala de usos de estas tecnologías, a su variedad y a la diversidad de criterios manejados para detallarlos y clasificarlos.

Algunos razonamientos que suelen surgir en las categorizaciones, bien de modo autónomo o mezclados, son los siguientes; primero, la clasificación de recursos tecnológicos manejados: computadoras, redes más o menos extensas de computadoras, sistemas de interconexión, soporte y formato de la información, plataformas, sistemas de administración de contenidos o de aprendizaje, aulas virtuales, etc. Segundo, el uso de aplicaciones e instrumentos que admiten la mezcla de recursos, como simulacros, materiales multimedia, tableros electrónicos, correo electrónico, registros de correo, conjuntos de noticias, mensajería breve, 
videoconferencia participativa, etc. En tercer lugar, la mayor o menor prolongación y riqueza de las interacciones que las tecnologías elegidas viabilizan. Cuarto, la etapa sincrónica o asincrónica de las participaciones; y finalmente, los objetivos y propósitos educativos que se buscan y las ideas supuestas o manifiestas del aprendizaje y de la enseñanza en las que se sostienen.

\section{Las TIC en el escenario actual}

Al parecer el sistema educativo no ha logrado estar al margen con los cambios que han surgido en el período digital, los cuales se ubicaron primero en la sociedad, y luego en la vida de los individuos y posteriormente en las instituciones educativas. Las TIC dejan de ser tan solo instrumentos tecnológicos en la educación, sino que pasan a ser parte de las competencias elementales a desarrollar en el lapso de la enseñanza-aprendizaje. Para Cueva (2020) se debe ir incluir instrumentos tecnológicos en el transcurso del proceso de enseñanza-aprendizaje, ya que es muy beneficio su uso; de este modo, queda en responsabilidad de los docentes manejarlos apropiadamente para avalar cambios importantes como consecuencia de su quehacer pedagógico.

Se reflexiona que hoy por hoy la inversión que se efectúa en equipos tecnológicos no está siendo aprovechada en su totalidad por los docentes; existen establecimientos formativos con áreas tecnológicas y vanguardistas que, en muchas veces son relegados o manejados ocasionalmente, careciendo de categorización, debido a que son concedidos a docentes con limitados conocimientos de los beneficios cognitivos que se dan en el proceso de enseñanza y aprendizaje al utilizar las TIC.

Los docentes en su necesidad de innovar examinan las TIC con el propósito de investigar, seleccionar, analizar, depurar, manejar y comunicar la información que se transformará en conocimiento, propiciando asimilación y afianzamiento de estos métodos e instrumentos tecnológicos para ser utilizados con sus estudiantes, que les admita evolucionar su entorno y desplegar un pensamiento crítico. Flores y Garrido (2019) indican que:

...la importancia que tienen las tecnologías en el aula y en las funciones docentes, originan un cambio sistemático en sus prácticas didácticas, donde (el profesor) asume un papel de gestionador de la información, dinamizador de los aprendizajes y una diversidad de particularidades que ruedan en torno a su perfil en la sociedad del conocimiento (p. 19).

Por tanto, se plantea nuevos roles y perfiles para los docentes, siendo primordialmente independientes, aspiradores, disciplinados, flexibles, planificadores, críticos e innovadores de su adecuada práctica educativa.

¿Pero qué ocurre cuando los docentes no se orientan al cambio y sus rasgos de personalidad quedan rezagadas a lo tradicional? Al respecto Garrido (2019) señala que los docentes, son quienes por recelo no rompen paradigmas y dificultan muchas veces los aprendizajes de los estudiantes y la aplicación de nuevas representaciones de trabajo interdisciplinario y de unificación de otros medios, como las TIC en los procesos de enseñanza. Razón por la cual, es imperioso reflexionar y procurar un cambio de actitud para interiorizar las innovaciones que se están promoviendo en la educación y admitan la posibilidad de integrar nuevas estrategias pedagógicas que respondan a los intereses, necesidades y estilos de aprendizaje de los estudiantes y visibilicen nuevas formas de gestionar el conocimiento. 
Los entornos virtuales en la educación: la innovación de los entornos tradicionales y la creación de nuevos ambientes de enseñanza

La tendencia educativa plantea "innovar" las estrategias, ambientes, recursos y métodos que propendan motivar al estudiante y optimizar la calidad de educación. En este ambiente de cambios profundos que el docente tiene el reto de reinventarse y reaprender en el camino a esta innovación, el cual se adhiere a la tecnología en su variedad de plataformas y aplicaciones web, postulando nuevos principios pedagógicos en un nuevo ambiente de instrucción (estimulación y conocimientos preliminares), donde es necesario establecer las aptitudes técnicas requeridas en el manejo de las nuevas TIC, en conjunción con una instrucción constructivista en detrimento del conductismo. (Chong y Marcillo, 2020).

Otra apreciación determina que la práctica docente en su ambiente pedagógico se debe percibir como un proceso basado en prácticas legítimas, abiertas y de contribución desde su planificación hasta su representación; es decir, que las clases se conviertan en un lugar de participación y el aprendizaje segeneraentreyparatodos. Actualmente existen diferentes guías acerca de los estándares de calidad aplicadas al "b-learnig" o al aprendizaje electrónico, existiendo seis organizaciones a nivel mundial (ADL, AICC, CEN, IEEE, IMS, ISO/ IEC) que se encargan de su aseguramiento y de las normativas para su implementación en ambientes escolares; pero es necesario alcanzar un punto fusionado hacia estándares comunes que regule el e-learning (Hilera y Hoya, 2010).

Para Bedoya y Muñoz (2019) la educación, obligada y demandada principalmente por los cambios sociales recurrentes, necesita de innovación y dinamización en sus esquemas, estrategias y mediaciones pedagógicas, a la luz de viabilizar y beneficiar su ejercicio, y con miras a evolucionar la sociedad y sus territorios.
En la actualidad, existen distintas metodologías educativas que modulan las tecnologías información y comunicación a los procesos de construcción del saber; dentro del campo de la tecnología educativa, los Entornos Virtuales de Aprendizaje han sido referenciados como una línea de innovación para la educación superior de ahora y del mañana, percibiendo múltiples paradigmas aproximados a los procesos de mediación pedagógica en la sociedad del conocimiento y en favor de la educación superior.

En la didáctica actual y presente, el aprendizaje es un proceso de aportación, de cooperación y colaboración grupal y comunicativo; en el cual los individuos despliegan el autoconocimiento, la responsabilidad individual y social, y aumentan su nivel de criticidad para analizar creadoramente; así como, para remediar inconvenientes y tomar decisiones. Es así como, entre los más importantes problemas encontrados hoy en día se encuentra el limitado conocimiento por parte de los docentes en el uso correcto de las metodologías y elementos tecnológicos para el diseño de sus clases; estas limitaciones en el manejo de estrategias de aprendizaje más prácticas en estos entornos dificultan el progreso sistémico de aprendizaje de los estudiantes.

El objetivo de esta investigación teórica se orientó hacia la necesidad de comprender la necesidad de utilizar efectivamente un EVA con un soporte teórico y procedimental que viabilicen el desarrollo de destrezas y nuevos principios de representación cognitiva que intervengan en el aprendizaje de quienes interactúan en estos entornos, transformándolos en un gran recurso pedagógico.

El propósito del estudio se centró en valorar una serie de estudios teóricos útiles para el diseño de procesos de enseñanza y aprendizaje en entornos virtuales, desde el enfoque del trabajo colaborativo 
el autoaprendizaje, y el rol dinámico del estudiante para adquirir conocimiento y desarrollar destrezas concernientes a la gestión del conocimiento mediante el uso de instrumentos relacionados con tecnología educativa (EVA) que le permitan dar respuesta a los requerimientos de los diversos modelos pedagógicos y modalidades de estudio como la presencial, semipresencial e hibrida, como beneficio para el desarrollo de sus competencias académicas, laborales y personales.

\section{MÉTODO}

El estudio es un esquema de revisión sistemática de literatura, y se considera como un análisis de investigación definida; su sustento metodológico le establece como una investigación cualitativa, la cual radicó en la recolección de información de los entornos virtuales como espacios de enseñanza y aprendizaje (EVEA). El nivel de investigación que comprobó la evidencia y credibilidad de la temática, manejo diferentes procesos como: preparatoria, descriptiva, interpretativa, cimentación y publicación, lo que permitió corroborar datos significativos y verídicos desarrollados anteriormente de forma empírica. La investigación se basó en artículos seleccionados por los investigadores de acuerdo con las reflexiones teóricas y metodológicas afines al tratamiento temático de los EVEA.

En la investigación se utilizó el modelo de investigación documental de Hoyos (2000), donde se destacan cinco fases: preparatoria, descriptiva, interpretativa por núcleo temático, de cimentación teórico global y de extensión y publicación (Figura 1).

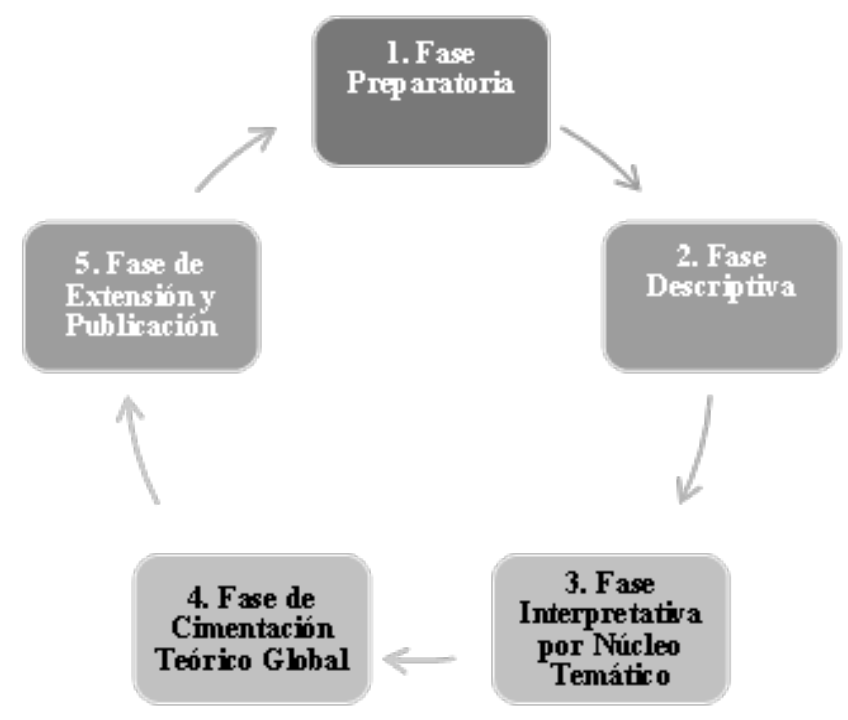

Figura 1. Fases de la investigación cualitativa del EVEA.

A continuación, se presentan cada una de estas fases para relacionarlas con la investigación:

\section{Fase 1 preparatoria}

Se describió el objeto de estudio, tema, lenguaje a manejar en el siguiente proceso: se contextualizaron los núcleos temáticos mediante los conocimientos y experiencias de los autores objeto de estudio; se definió el tema, las técnicas y la base de datos académica de donde procedió la fuente documental (Tabla 1). 
Tabla 1. Fase preparatoria (selección documental).

\begin{tabular}{|c|c|c|c|c|c|}
\hline No & & es de datos académicas & $\begin{array}{l}\text { Publicaciones } \\
\text { científicas y } \\
\text { tecnológicas }\end{array}$ & Documentación & Tiempo \\
\hline 1 & $\begin{array}{l}\text { Redalyc: es } \\
\text { un sistema de } \\
\text { información } \\
\text { científica }\end{array}$ & $\begin{array}{l}\text { Artículo Científico de la Revista } \\
\text { Ciencias Holguín } \\
\text { https://www.redalyc.org/articulo. } \\
\text { oa?id=181542152006 }\end{array}$ & $\begin{array}{l}\text { Identificación de } \\
\text { entornos virtuales de } \\
\text { enseñanza aprendizaje } \\
\text { (EVEA) en la } \\
\text { educación virtual }\end{array}$ & $\begin{array}{l}\text { La selección de la } \\
\text { documentación } \\
\text { relacionada se realiza por } \\
\text { medio de las palabras } \\
\text { clave EVEA en la } \\
\text { enseñanza y aprendizaje. }\end{array}$ & 2015 \\
\hline 2 & & $\begin{array}{l}\text { Artículo Científico de la Revista } \\
\text { Pixel-Bit. Medios y Educación } \\
\text { https://www.redalyc.org/ } \\
\text { pdf/368/36849882009.pdf }\end{array}$ & $\begin{array}{l}\text { La representación social } \\
\text { en entornos virtuales } \\
3 \mathrm{~d} \text { : análisis a partir } \\
\text { de una práctica en la } \\
\text { universidad }\end{array}$ & & 2017 \\
\hline 3 & $\begin{array}{l}\text { Scielo: es un } \\
\text { sistema de } \\
\text { información } \\
\text { científica }\end{array}$ & $\begin{array}{l}\text { Artículo Científico de la Revista } \\
\text { Revista Electrónica Educare } \\
\text { https://www.scielo.sa.cr/ } \\
\text { scielo.php?pid=S1409- } \\
\text { 42582018000100364\&script=sci_ } \\
\text { arttext }\end{array}$ & $\begin{array}{l}\text { Entornos virtuales de } \\
\text { aprendizaje complejos } \\
\text { e innovadores: Una } \\
\text { práctica de espacio } \\
\text { participativo desde el } \\
\text { paradigma emergente }\end{array}$ & & 2018 \\
\hline 4 & & $\begin{array}{l}\text { Artículo Científico de la Revista } \\
\text { RIDE. Rev. Iberoam. Investig. } \\
\text { Desarro } \\
\text { http://www.scielo.org.mx/ } \\
\text { scielo.php?pid=S2007- } \\
\text { 74672019000200025\&script=sci_ } \\
\text { arttext }\end{array}$ & $\begin{array}{l}\text { La formación de } \\
\text { docentes normalistas: } \\
\text { De la tradición } \\
\text { pedagógica a los } \\
\text { entornos virtuales de } \\
\text { aprendizaje }\end{array}$ & & 2018 \\
\hline 6 & $\begin{array}{l}\text { Dialnet: bases } \\
\text { de datos } \\
\text { referenciales y } \\
\text { multidisciplinares } \\
\text { disponibles en } \\
\text { español. Además, } \\
\text { recoge gran } \\
\text { parte de la } \\
\text { información } \\
\text { científica. }\end{array}$ & $\begin{array}{l}\text { Artículo Científico de la Revista } \\
\text { Horizontes Pedagógicos } \\
\text { file://C:/Users/59398/Downloads/ } \\
\text { Dialnet-ModelosDeAprendizaj } \\
\text { eVirtualesYPresencialesEnLect } \\
\text { oe-7415989.pdf } \\
\text { Acta - Comité Latinoamericano de } \\
\text { Matemática } \\
\text { file://C:/Users/59398/ } \\
\text { Downloads/Dialnet- } \\
\text { AnalisisDocumental-7659491.pdf }\end{array}$ & $\begin{array}{l}\text { Estudios documentales: } \\
\text { importancia de los } \\
\text { entornos virtuales en } \\
\text { los métodos educativos } \\
\text { en el nivel superior. }\end{array}$ & & 2020 \\
\hline
\end{tabular}

Fuente: Bases bibliográficas 


\section{Fase 2 Descriptiva.}

Se realizó un análisis descriptivo para identificar los diferentes tipos de estudio que se han realizado, los referentes disciplinares, teóricos, poblaciones, delimitaciones, autores y metodologías que se han aplicado. De la revisión minuciosa de cada unidad de análisis se genera una ficha descriptiva (Tabla 2).

Tabla 2. Fase descriptiva

\begin{tabular}{ll}
\hline \multicolumn{1}{c}{ Categorías } & \multicolumn{1}{c}{ Categorías simplificadas } \\
\hline Qué es (EVEA) & Conceptualización del modelo \\
Conceptualización & \\
Características pedagógicas & Requisitos de implementación académica \\
Uso de aulas virtuales. & \\
Lineamientos tecnológicos y pedagógicos & Estrategias innovadoras \\
\hline
\end{tabular}

Fuente: Hoyos (2000)

Al mismo tiempo, en la revisión documental se agruparon las palabras claves y los párrafos, identificando las palabras frecuentes que refuerzan el estudio y se resaltan en diferentes párrafos para relacionarlos a cada categoría. Los documentos fueron analizados cualitativamente por N-VIVO, validando las palabras frecuentes que definen las categorías de análisis.

\section{Fase 3. Interpretativa por núcleo temático.}

Se revisó cada documento para elegir los conceptos, autores y teorías que respaldan los resultados de la presente investigación de los entornos virtuales de enseñanza aprendizaje (EVEA), más aún, los procesos y procedimientos que se evidencian en la lectura de las fuentes de autores representativos seleccionados en la fase preparatoria que sustenten las categorías establecidas en la investigación (Tabla 3).

Tabla 3. Interpretativa por núcleo temático.

\begin{tabular}{|c|c|c|c|}
\hline Categoría & Autor(es) (año) & Concepto & Justificación \\
\hline Conceptualización & $\begin{array}{l}\text { Guaña - Moya, Edison } \\
\text { Javier (2015) }\end{array}$ & $\begin{array}{l}\text { Su autor argumenta que los EVEA } \\
\text { sirve para lograr una dinámica en la } \\
\text { diversidad de recursos } \\
\text { didáctico-tecnológicos, así como la } \\
\text { cooperación continúa de los } \\
\text { participantes y sus entornos } \\
\text { con tendencia a eliminar sus } \\
\text { deficiencias y que la formación de } \\
\text { los estudiantes. }\end{array}$ & $\begin{array}{l}\text { Es importante que el docente } \\
\text { adquiera aptitudes tecnológicas } \\
\text { en suformación, el modelo EVEA } \\
\text { identificando los conocimientos } \\
\text { que debe dominar para integrar } \\
\text { las TIC eficazmente en el aula con } \\
\text { el objetivo de lograr resultados } \\
\text { importantes en el aprendizaje de } \\
\text { los estudiantes. }\end{array}$ \\
\hline
\end{tabular}

Fuente: Elaboración propia 


\section{Fase 4. Cimentación teórica global}

Se interpretó cada núcleo temático para identificar tendencias, logros, vacíos, limitaciones, dificultades de la temática estudiada, con la intención de presentar el estado actual de la investigación de forma global para orientar nuevas líneas de investigación.

\section{Fase 5. Extensión y publicación}

Se encontró que la publicación de este artículo era la mejor forma de dar a conocer el conocimiento adquirido sobre los EVA a la colectividad académica o científica.

\section{RESULTADOS}

Una vez recopilados los documentos aplicando las fases preparatoria, descriptiva, interpretativa, cimentación y extensión se obtuvo los resultados de acuerdo con las categorías diseñadas y los parámetros determinados para el análisis, utilizando el software N-VIVO.

\section{Preparatoria documental}

En este punto en el programa, en N-VIVO, se identificaron las palabras, relaciones, criterios, y estadísticas en los documentos trabajados (Tabla 1). Se sometieron al análisis en el software a los documentos que se seleccionaron por ser afines a la temática y objetivos del estudio de investigación. Los documentos se categorizan por título, autor(es), año y tipo de contenido referente al tema de investigación (Tabla 4).

Tabla 4. Clasificación Preparatoria documental.

\begin{tabular}{ccc}
\hline Unidad de análisis & Frecuencia & Porcentaje \\
\hline Artículos & 4 & $67 \%$ \\
Libros & 1 & $17 \%$ \\
Tesis & 1 & $17 \%$ \\
Total & 6 & $100 \%$ \\
\hline
\end{tabular}

Fuente: Bases bibliográficas y fuentes documentales seleccionadas.

La distribución porcentual de las unidades de análisis (Tabla 4 y Figura 2) establece que el 67\% de las fuentes documentales pertenecen a artículos; mientras el $17 \%$ son trabajos de tesis; seguido de un $16 \%$ de libros. Este resultado determina que el estudio se centró en artículos como fuentes documentales principales. 


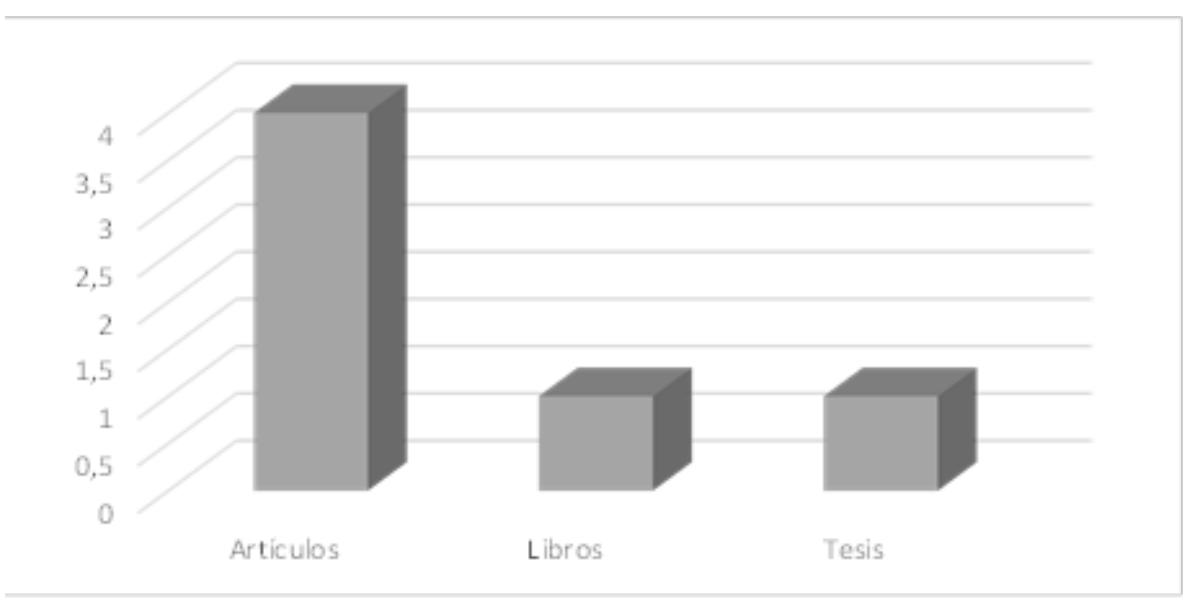

Figura 2. Clasificación Preparatoria documental.

\section{Descriptiva}

Cada país establece sus políticas educativas de acuerdo con la cultura, el tipo de sociedad, entre otros, lo cual determina la aplicabilidad de las TIC dentro de un modelo educativo, ya sea dentro de educación virtual y-o presencial. El número de documentos publicados revisados con conocimiento y procedimientos para la implementación de la temática de los entornos virtuales en la educación demuestra la importancia del estudio de esta temática (Tabla 5).

Tabla 5. Unidad de análisis por país.

\begin{tabular}{ccc}
\hline País & Frecuencia & Porcentaje \\
\hline España & 3 & $50 \%$ \\
Chile & 2 & $33 \%$ \\
Colombia & 1 & $17 \%$ \\
Total & 6 & $100 \%$ \\
\hline
\end{tabular}

Fuente: Bases bibliográficas y fuentes documentales seleccionadas.

El estudio (Figura 3) determinó que España es el país de mayor producción académica de este tema, seguido de Chile y Colombia; razón por la cual se seleccionó en esta proporción los estudios a ser considerandos en el desarrollo de la investigación. 


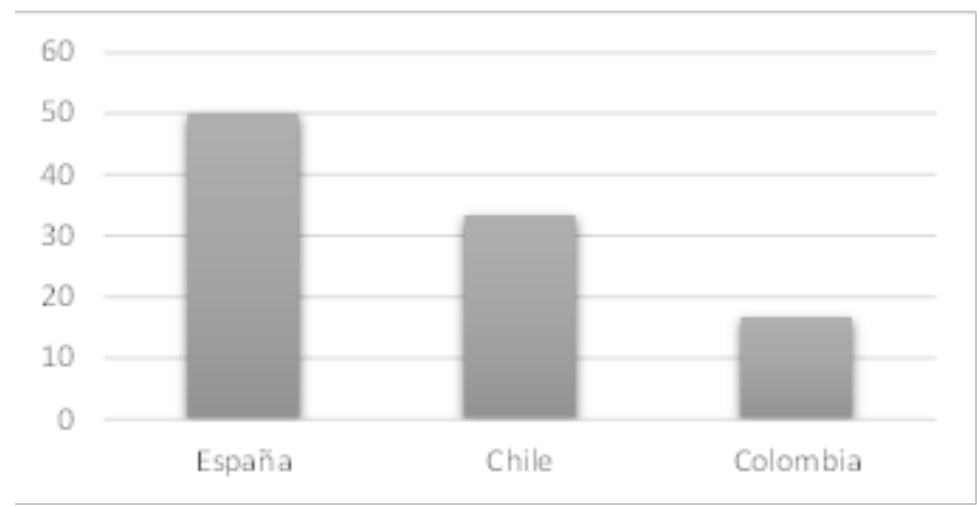

Figura 3. Unidad de análisis por país.

El estudio determina que hasta hace cinco documental se visualiza en la muestra de años atrás la investigación académica de los entornos virtuales en la educación ha aumentado documentos, que el mas de la mitad de los artículos significativamente (Tabla 6). De la selección son publicados en estos últimos años.

Tabla 5. Unidad de análisis por país.

\begin{tabular}{ccc}
\hline Años & Frecuencia & Porcentaje \\
\hline 2015 & 1 & 17 \\
2017 & 1 & 17 \\
2018 & 2 & 33 \\
2019 & 1 & 17 \\
2020 & 1 & 17 \\
Total & 6 & $100 \%$ \\
\hline
\end{tabular}

La tendencia va de aumento sobre la debería al aumento de oferta en la educación virtual conceptualización y estudios del modelo de o el manejo de intervenciones (Figura 4). investigación de los entornos virtuales, esto se

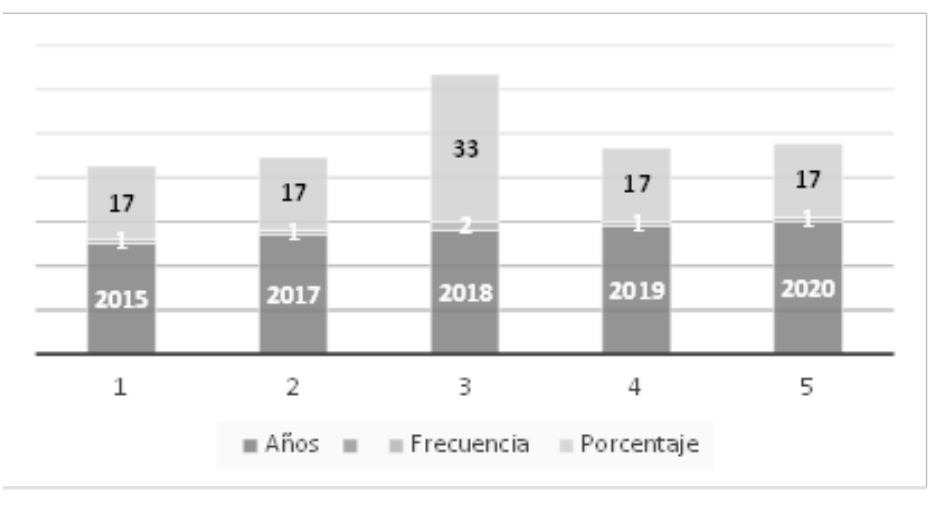

Figura 4. Fuentes documentales analizados por año de publicación. 


\section{Interpretativa por núcleo temático}

De acuerdo con las categorías planteadas producto de los parámetros establecidos para el análisis a través del software N-VIVO, se evidencia, que los documentos asociados, reúnen la información necesaria sobre los principios del EVEA con respecto al conocimiento pedagógico disciplinar, el conocimiento tecnológico pedagógico, y la relación entre conocimiento tecnológico disciplinar.

\section{Cimentación teórica global}

\section{a. Precisar propósito de análisis}

Los documentos, fueron sometidos al análisis cualitativo mediante el programa N-VIVO, teniendo en cuenta la lectura de cada uno y selección de palabras clave que refuerza el estudio de investigación; utilizando este se identificó las palabras frecuentes que definen las categorías de análisis. La mezcla de las palabras y sus significados permitió constituir frases u oraciones, y la suma de las distintas palabras, una expresión fija, como consecuencia de esta acción se encontró un significado propio y específico. Los significados de las palabras se manejan para ligarla con la capacidad del habla, la aptitud en la oratoria, la caracterización escrita de la expresión oral o lo expresado por otra persona.

\section{Extensión y publicación}

\section{a. Construcción de categorías}

Las categorías definidas durante la investigación fueron: conceptualización del modelo; lineamientos pedagógicos, tecnológicos y requisitos de implementación académica. Posteriormente, se validaron las categorías, mediante la identificación por frecuencias de las palabras que definen los entornos virtuales de enseñanza aprendizaje (EVEA), de acuerdo con el rastreo realizado desde software utilizado.

\section{b. Procesamiento y análisis de los datos}

Definidas las categorías de análisis, cada una de ellas fueron sometidas a un seguimiento de las frecuencias de palabras identificadas en cada uno de los artículos, con la señalización de los aportes identificados se pudo dar explicación de las finalidades de cada una de las propuestas que se sometieron al estudio de tipo cualitativo acordes a la investigación.

La selección de cada uno de los artículos correspondió a la búsqueda de palabras claves empleadas durante el desarrollo de la investigación. Dichas palabras se enmarcaron en cada uno de los artículos encontrados y permitieron el desarrollo del análisis. Es por eso, que, las palabras empleadas durante la búsqueda de información fueron: Entornos virtuales, enseñanza - aprendizaje, EVA, EVEA, metodología, conocimiento tecnológico, pedagogía, educación virtual, competencias, TIC, lo cual limito el universo de búsqueda.

\section{Discusión}

Estos resultados delimitan la importancia de crear procesos pedagógicos en los docentes para la ejecución de las herramientas tecnológicas en el aula como eje integral de los métodos de enseñanza del discernimiento y el manejo de entornos virtuales. Por otro lado, la investigación sobre lineamientos pedagógicos, tecnológicos, resalta que es transcendental relacionarlos a los elementos conceptuales de una disciplina para ser ejecutado y enseñando en un programa explícito, instaurando correlación inmediata entre el discernimiento tecnológico y pedagógico y, la comprensión pedagógica disciplinar. Por su parte 
los demás documentos analizados y asociados a conceptualización del modelo, congrega la información necesaria para fortificar el manejo y aumento de entornos virtuales en la enseñanza aprendizaje.

\section{CONCLUSIONES}

En la búsqueda bibliográfica se pudo evidenciar que existe la necesidad de fomentar el uso de Entornos Virtuales de Enseñanza-Aprendizaje (EVAE) en la educación en todos sus niveles. De acuerdo al objetivo que se planteó sobre la utilización de EVEA, se analizó información sobre aspectos teóricos que viabilicen el progreso de destrezas de la virtualidad como e-learning, el b-learning, y el m-learning, lo que permitió establecer la dinámica que se plantea en la educación y los proceso de formación profesional con entornos virtuales de enseñanza aprendizaje, cuya utilización permitirá un mejor desarrollo del aprendizaje individual y colaborativo en estos entornos, así como también la auto - construcción de nuevos conocimientos.

Los resultados obtenidos con el procesamiento y análisis de los datos cualitativos en el programa $\mathrm{N}$-VIVO, determinó la necesidad de incorporar y reforzar el estudio de los Entornos Virtuales de Enseñanza Aprendizaje, ya que existe una gran cantidad de estudios y trabajos científicos afines a este estudio y que trata sobre los EVEA's, la metodología activa, el conocimiento tecnológico, la pedagogía del contenido, las TIC, la educación virtual, las competencias, entre otros; para lo cual es necesario puntualizar el universo de búsqueda para una mejor comprensión de los procesos educativos virtuales; y como esta ayuda a los docentes en su proceso de enseñanza diaria y continua.

Finalmente, existe limitaciones en la formación docente en los procesos de formación en EVEA; además, en la literatura se establece que no existe una preparación preliminar para que los estudiantes consigan auto educarse en el uso de estas nuevas tecnologías; así como también, en su uso en actividades cooperativas y colaborativa dentro de los entornos virtuales, es por esto, que es necesario una preparación y motivación por parte de los docentes que les permita construir propuestas didácticas integradoras que promuevan nuevas formas de enseñar y de aprender a los educandos a través de la integración de las TIC, para transformar los procesos de formación vinculadas a los objetivo educativo del nivel superior.

\section{REFERENCIAS}

Abarca, A., Alpízar, F., Sibaja, G., y Rojas, C. (2012). El análisis de contenido. Capítulo VI. En. Técnicas Cualitativas de Investigación. UCR. Costa Rica, 39-45.

Bedoya, G. V., y Muñoz, O. J. (2019). Alternativas emergentes de mediación pedagógica en la sociedad del conocimiento para la educación superior: tecnología educativa, entornos virtuales de aprendizaje y posibles paradigmas globales de innovación aplicables al contexto colombiano. Medellín: Universidad Nacional Abierta y az Distancia-UNAD.

Cedeño, R. E. (2019). Entor nos virtuales de aprendizaje y su rol innovador en el proceso de enseñanza. ReHuSo, 119-127.

Chong, B. P., \& Marcillo, G. C. (2020). Estrategias pedagógicas innovadoras en entornos virtuales de aprendizaje . Dominio de las Ciencias, 56-77.

Cueva, G. D. (2020). La tecnología educativa en tiempos de crisis. Scielo, 341-348.

Fernández, P. S., y Arroyo, V. Z. (2018). Entornos virtuales de aprendizaje en comunidades de práctica de docentes universitarios del Ecuador. Ensayos Pedagógicos, 185-200.

Flores, C. D., y Garrido, S. J. (2019). Competencias digitales para los nuevos escenarios de aprendizaje en el contexto universitario . Scientific, 44-61. 
Hilera, G. J., y Hoya, Marín, R. (2010). Estándares e-learning: guia de consulta. España: Universidad de Alcalá.

Hoyos, C. (2000). Un modelo para investigación documental. Guía teórico-práctica sobre construcción de Estados del Arte con importantes reflexiones sobre la investigación. Señal Editora., 9-25.

León, C. F., y Moracén, C. J. (2016). Los entornos virtuales de enseñanza-aprendizaje. Un contenido a sistematizar en el proceso de superación profesional del docente. Santiago, 292-307.

Nóbile, C. I., y Luna, Á. E. (2015). os Entornos Virtuales de Enseñanza y Aprendizaje en la Universidad Nacional de La Plata. Una aproximación a los usos y opiniones de los estudiante. InnoEduca, 3-14.
Sánchez, A. B. (2010). Los Entornos Virtuales como Espacios de Enseñanza y Aprendizaje. Una Perspectiva psicoeducativa para su caracterización y análisis. Revista Mexicana de Investigación Educativa, 163-184.

Sánchez, A. B., y Salvador, C. C. (2010). Los entornos virtuales como espacios de enseñanza y aprendizaje. Una perspectiva psicoeducativa para su caracterización y análisis. Revista mexicana de investigación educativa, 163-183. 